\title{
Impedance Issues in the CERN SPS
}

\section{T.Linnecar}

\begin{abstract}
The future use of the CERN SPS accelerator as injector for the Large Hadron Collider, LHC, and the possible use of the SPS as a neutrino source for the Gran Sasso experiment are pushing the maximum intensity requirements of the accelerator much higher than achieved up to now. At the same time the requirements on beam quality are becoming far more stringent. The SPS machine, built in the 70's, is not a "smooth" machine. It contains many discontinuities in vacuum chamber cross-section and many cavity-like objects, as well as the 5 separate RF systems at present installed. All these lead to a high impedance, seen by the beam, spread over a wide frequency range. As a result there is a constant fight against instabilities, both single and multi bunch, as the intensity increases. A programme of studies is under way in the SPS to identify, reduce, and remove where possible the sources of these impedances.
\end{abstract}

Presented at the Workshop on Instabilities of High Intensity Hadron Beams in Rings, Brookhaven National Laboratory, 28 June - 1 July 1999

Geneva, Switzerland

20th July 1999 


\section{Contents}

1 Introduction $\quad 1$

2 Future beam requirements for the SPS $\quad 1$

3 What type of problems do we expect? $\quad 2$

4 Identifying the sources 3

4.1 Impedances leading to a single bunch instability . . . . . . . . . . . . . . . . . . 3

4.2 Impedances leading to multi-bunch instabilities . . . . . . . . . . . . . . 5

5 Reducing the machine impedance $\quad 5$

5.1 Elements with high $\mathrm{R}_{\mathrm{sh}} / \mathrm{Q} \ldots \ldots \ldots \ldots \ldots \ldots$

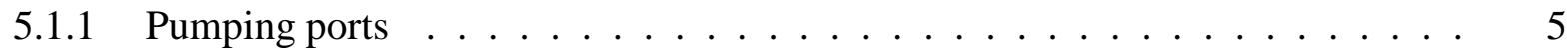

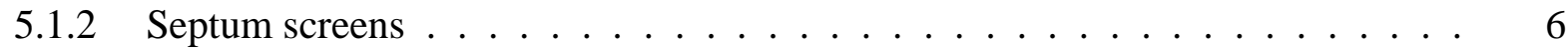

5.2 Elements with high $\mathrm{R}_{\mathrm{sh}} \ldots \ldots \ldots \ldots \ldots \ldots \ldots$

$\begin{array}{llr}6 & \text { Other means of controlling the instabilities } & 7\end{array}$

7 Conclusion $\quad 9$ 


\section{Introduction}

To fulfil the different fixed target physics requirements the total intensity of the CERN SPS accelerator has increased from its design value of $10^{13}$ protons/pulse, achieved soon after commisioning in 1976, to its present peak value of $4.84 \times 10^{13}$ attained in 1997 . In the early 80 's, the SPS became the Spp̄S collider where 6 proton bunches were accelerated and then collided with 6 antiproton bunches. The single bunch intensity was increased during this time from the $10^{10}$ in fixed target operation to $1.6 \times 10^{11}$. To obtain these total and single bunch intensity increases a continuing battle has had to be waged against instabilities, leading to many modifications and upgrades to the machine hardware. Here are a few examples. Already in the first two years of operation the impedance of two higher order modes, one transverse at $460 \mathrm{MHz}$ and the other longitudinal at $628 \mathrm{MHz}$ [1] in the main RF cavities, were identified as causing instability and intensity limitation and had to be passively damped. When preparing for $S p \bar{p} S$, bunch intensities were limited when crossing transition by both a longitudinal negative mass instability and a transverse head-tail instability to $2 \times 10^{10}$ protons [2]. This limitation was removed by raising the injection energy to $\mathrm{E}=26 \mathrm{GeV}$, above transition, $\gamma_{t r}=23.4$. Single turn RF feedback has been required for the main $200 \mathrm{MHz}$ travelling wave RF system to raise intensities above $3 \times 10^{13}$. This type of feedback has also had to be added in addition to the strong short delay RF feedback around the 352 $\mathrm{MHz}$ superconducting cavities used for lepton acceleration, but also seen by the intense proton beam. Together they reduce the impedance at the synchrotron satellites around each revolution frequency line from many $\mathrm{G} \Omega$ to $\sim 100 \mathrm{k} \Omega$ per cavity.

Future uses of the SPS require much higher intensities. At the same time much improved beam quality is demanded. The presence or not of instabilities will clearly be a dominant factor in the success of our programme. Identification and control of the different impedance sources in the machine is and will remain a very hot issue.

\section{Future beam requirements for the SPS}

The SPS machine is now being prepared for use as injector for the Large Hadron Collider (LHC) [3], at present under construction at CERN and which will come into operation in 2005. To obtain the highest luminosities in the LHC, the SPS must accelerate beams with single bunch intensities similar to that in Sp $\bar{p} S$ operation, $\mathrm{N}=1.1 \times 10^{11}$, and batch intensities much higher than seen before in the SPS, even though the total intensity will be lower than the record value due to the incomplete filling of the ring, $2.4 \times 10^{13}$ protons in $3 / 11$ of the ring. These performances must be achieved with small longitudinal and transverse emittances, both for luminosity reasons, but also in the longitudinal case to ease transfer to the LHC where beam losses at injection must be kept very small [4].

\begin{tabular}{|c|c|c|c|c|c|c|}
\hline $\begin{array}{l}\text { Operating } \\
\text { Mode }\end{array}$ & $\begin{array}{c}\text { Energy } \\
\text { inj., top } \\
(\mathrm{GeV})\end{array}$ & $\begin{array}{c}\text { Total } \\
\text { intensity } \\
\left(\times 10^{12}\right)\end{array}$ & $\begin{array}{c}\text { Bunch } \\
\text { intensity } \\
\left(\times 10^{10}\right)\end{array}$ & $\begin{array}{c}\text { Batch } \\
\text { current } \\
\text { (A) }\end{array}$ & $\begin{array}{c}\varepsilon_{l} \\
\text { inj., top } \\
(\mathrm{eVs})\end{array}$ & $\begin{array}{c}\varepsilon_{H, V} \\
\text { top } \\
(\mu m)\end{array}$ \\
\hline $\begin{array}{c}\text { Spp̄S collider } \\
\text { (past) }\end{array}$ & 26,315 & 0.2 & 20 & & $0.6,0.8$ & $2.75,2.75$ \\
\hline $\begin{array}{c}\text { Fixed target } \\
\text { (present) }\end{array}$ & 14,450 & 48.4 & 1 & 0.33 & $0.2,2$ & 10,7 \\
\hline $\begin{array}{c}\text { SPS for LHC } \\
\text { (project) }\end{array}$ & 26,450 & 24 & 11-17 & $0.7-1.1$ & $0.35,0.6-1$ & $\begin{array}{c}3.5,3.5 \\
(3.0 \text { inj.) }\end{array}$ \\
\hline $\begin{array}{c}\text { Gran Sasso } \\
\text { (possible) }\end{array}$ & 14,400 & $\geq 70$ & 2 & 0.5 & $0.2, \leq 2$ & 10,10 \\
\hline
\end{tabular}

Table 1: Parameters for present and future operation of the SPS 
Table 1 gives various parameters for this mode of operation, and also for the present fixed target physics operation and the past $S p \bar{p} S$ operation. The tight emittance budget for LHC beams in the SPS is evident. To allow going to the higher value of longitudinal emittance at extraction, $\varepsilon_{l}=1 \mathrm{eVs}$, it will be necessary to install a separate RF system at $200 \mathrm{MHz}$ in the LHC for capture [5]. Note also that present fixed target operation, with its lower batch current, has a far higher emittance at extraction than can be tolerated for LHC injection. This tight specification for the beam is supplemented by the further demand from the project to send a neutrino beam to Gran Sasso, Italy. This project requires maximising the total number of protons that can be produced by the SPS in a given operation period, say 200 days [6]. The optimisation of the accelerator chain is under study, but one parameter is clearly the maximum intensity per cycle that can be accelerated in the SPS. A desirable increase to $\geq 7 \times 10^{13}$ from the present maximum of $4.84 \times 10^{13}$ is hoped for. Although the emittances are less of a problem here, considerable care must be taken to prevent micro-losses during the acceleration cycle and in the extraction channel.

\section{What type of problems do we expect?}

The microwave instability is the single bunch instability of concern in the longitudinal plane. It was conjectured as early as 1976 [1] that the large number of discontinuities in the machine vacuum chamber could be the cause of the microwave instability responsible for blow-up during the debunching and recapture process at $10 \mathrm{GeV}$ in the SPS. The problem was solved at that time by installing a $200 \mathrm{MHz}$ RF bunching system in the PS (the SPS injector) and injecting bunch into bucket.

In $S p \bar{p} S$, the microwave instability limited the bunch intensity at injection at $26 \mathrm{GeV}$, the limit being raised by installing a $100 \mathrm{MHz}$ RF capture system in the SPS to allow transfer of longer bunches (5.0ns). It is believed from our present knowledge of the impedances responsible for this instability (see below) that the nominal LHC bunch of $4 \mathrm{~ns}, 0.35 \mathrm{eVs}$, and $1.1 \times 10^{11}$ protons, may be unstable. Rather than using a new RF system this time to reduce the danger, more radically the problem will be attacked at the source.

Multibunch instabilities in the longitudinal plane have been observed and studied on the operational fixed target beams and on batch type beams in machine study periods. Some conclusions from these studies can be drawn:

- The beam is already unstable at $4 \times 10^{12}$, becoming more and more unstable towards higher energies.

- The emittance at $445 \mathrm{GeV}$ for $4 \times 10^{13}$ is $\sim 2 \mathrm{eVs}$ for the transfer voltage needed for LHC injection, but can be lower with voltage programming.

- Bunch intensity seems to be the dominant parameter for emittance blow-up. However some influence from the large gap between the batches suggests a wakefield covering several bunches.

The first point can be explained by an analysis of the threshold intensity through the cycle. The threshold curves for the normal operation cycle and various resonator frequencies are shown in Fig. 1 taken from Ref.[7]. Whatever the source frequency, the threshold decreases significantly as energy increases. The spectrum sometimes shows specific lines growing but in general a broad spectrum filling the space between the bunch spacing frequency lines is seen.

In the transverse plane the head-tail instability, which is of greatest concern for single bunches, can be adequately controlled by careful chromaticity adjustment for the lowest modes. For the higher modes the frequency spread in the beam should be sufficiently large to give adequate Landau damping. The transverse mode coupling instability has not been observed with protons. Multi-bunch modes at high mode numbers are not at present observed, several transverse HOMs in the different RF cavities having been already damped. With increasing intensity this may no longer be the case. The resistive wall instability is of concern. For a given mode the risetime of this instability is modified by the batch structure of the beam, as is the mode number having the highest growth rate [8]. 


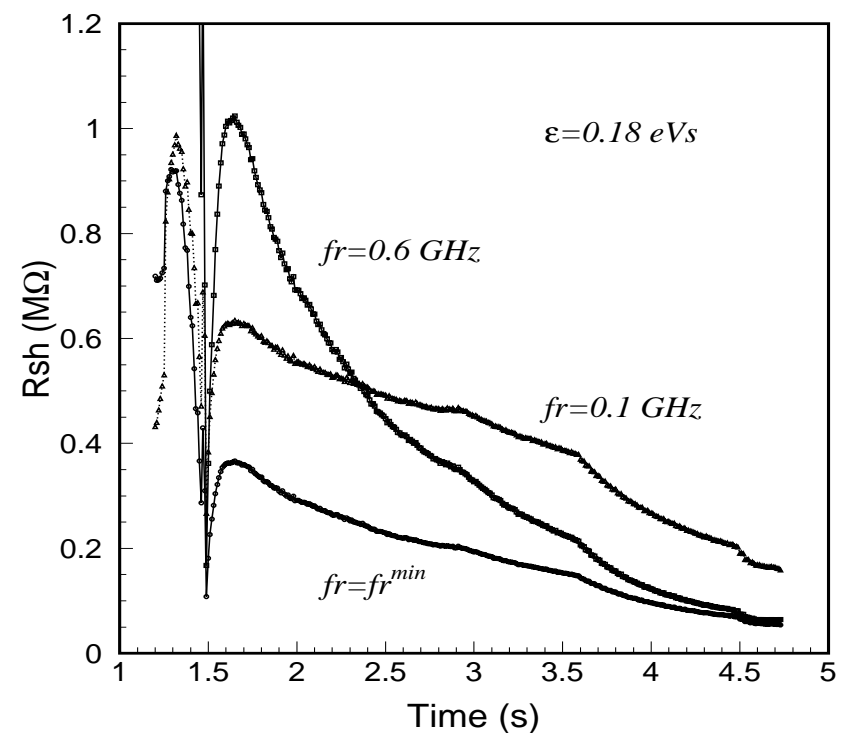

Figure 1: Coupled bunch instability threshold during normal operation in fixed target cycle for a beam intensity $4.2 \times 10^{13}$ and different resonant frequencies $f_{r}$.

\section{Identifying the sources}

\subsection{Impedances leading to a single bunch instability}

The microwave instability threshold has been determined in the past and more recently [9] by measuring the variation in captured bunch length as a function of injected intensity. The threshold can also be found by observing the high frequency signals generated. From the threshold intensity the parameters for a broad-band impedance model can be defined and this is often used as a machine parameter for scaling purposes. Estimations of this impedance depend on the spectral bandwidth of the particular bunch available for the measurement. The low-frequency inductive part can also allow this impedance to be measured and this can be found from bunch lengthening or by observation of the change in debunching time as a function of intensity [10].

However the the broad-band model is far from reality, making prediction for future operation modes from scaling very difficult. As is now clear, a more realistic model consists of a number of resonant peaks. Attempts to determine a model by making an inventory of the different elements in the machine and computing the longitudinal impedance have been made [11] as well. We have developed a technique based on the measurement of the spectrum of unstable bunch modes to measure the fine structure of the machine impedance [12] and determine the dominant impedances for the model. Here we outline the procedure used and give the results.

Single, high-intensity proton bunches are injected into the machine with RF off and the spectrum is observed during very slow debunching. If the bunch intensity is above threshold then the different resonant impedances in the machine lead to a modulation of the line density at the resonant frequencies. The debunching time, $t_{\mathrm{deb}}$, must be slow enough that the modulation grows and saturates before the bunch length has significantly increased. This is a function of the machine parameters, slip factor $\eta$, and the maximum momentum spread $\Delta \mathrm{p} / \mathrm{p}$ in the bunch. The instability reaches some maximum modulation amplitude which is recorded as a function of frequency. At each frequency point, the amplitude from many bunches is measured and averaged. The spectrum of an unstable bunch mode associated with a given narrow band impedance is centred at the resonant frequency and has a spectrum width given by the bunch distribution, proportional to $1 / \tau_{b}, \tau_{b}$ being the bunch length. Hence it is interesting to use very 
long bunches which then allow the fine structure of the machine impedance to be resolved. While exact analytical formulae for saturation are unknown, numerical simulation [9] shows that for a narrow band impedance source with bandwidth $\Delta f_{r}<1 / \tau_{b}$, the maximum amplitude is a function of the $\mathrm{R}_{\mathrm{sh}} / \mathrm{Q}$ of the cavity and $\mathrm{N}$.

In our experiment the machine and bunch parameter were as in Table 2:

\begin{tabular}{|c|c|c|c|c|}
\hline $\mathrm{E}$ & $\eta$ & $\tau_{b}$ & $\varepsilon_{l}$ & $\mathrm{t}_{\mathrm{deb}}$ \\
\hline $26 \mathrm{GeV}$ & $5.257 \times 10^{-4}$ & $25 \mathrm{~ns}$ & $0.25 \mathrm{eVs}$ & $80 \mathrm{~ms}$ \\
\hline
\end{tabular}

Table 2: Experimental parameters for measuring the spectrum of unstable bunches.

We used two techniques to analyse the data, which was obtained from a wide-band wall-current monitor. In the first one we used a spectrum analyser with a bandwidth of $3 \mathrm{MHz}$ as a receiver and scanned the range from $100 \mathrm{MHz}$ to $4 \mathrm{GHz}$. The second technique uses a digital oscilloscope to acquire the data in time domain, a Fourier transform giving the spectrum. This is much faster but is limited in any case to $2 \mathrm{GHz}$ by the maximum sampling frequency. The results obtained by the first technique are shown in Fig.2, where numerous peaks corresponding to the different impedance in the machine can be seen.

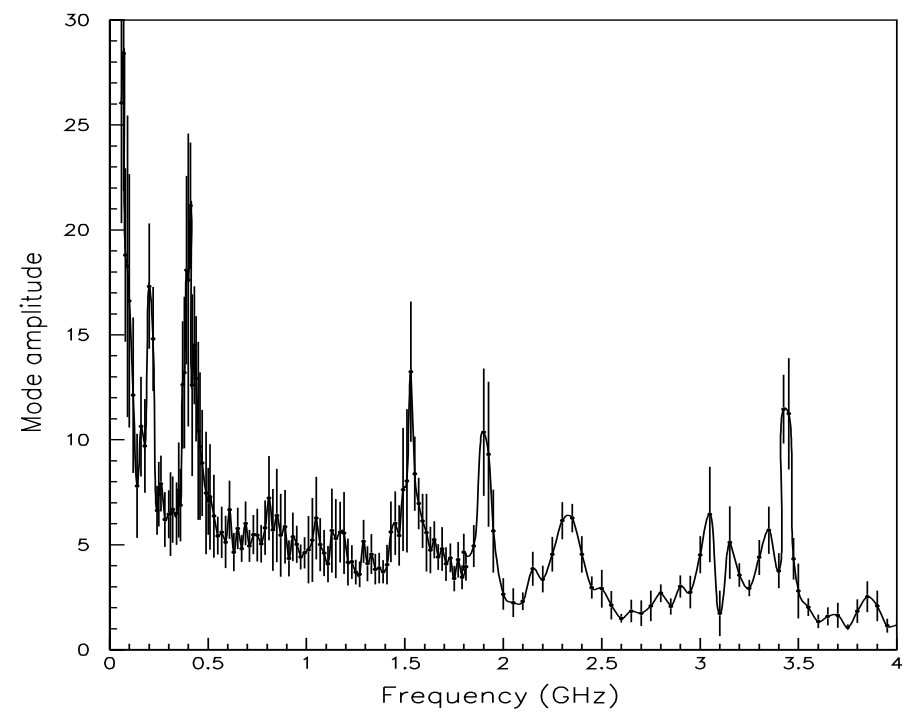

Figure 2: Measured spectrum of unstable bunches

We have succeeded in identifying most of the peaks in the figure. The peaks at $200 \mathrm{MHz}$ and 800 $\mathrm{MHz}$ are the travelling wave RF fundamental modes and the peak at $400 \mathrm{MHz}$ is due to the cavities formed by the vacuum chambers containing the extraction septa. All the peaks above $1.4 \mathrm{GHz}$ can be explained by the resonances of the 800 or so cavity-like vacuum ports. All these latter accidental cavities contain damping resistors, which is important for coupled bunch instabilities but which does not change the $\mathrm{R}_{\mathrm{sh}} / \mathrm{Q}$.

We have used bunch lengthening measurements to check our new impedance model which contains the four sets of elements described above with $R_{\mathrm{sh}} / \mathrm{Q}$ and $\mathrm{Q}$ values found from calculation or laboratory experiment. Results of simulations of bunch lengthening using this model can be compared with experimental results and show very close agreement. It seems that we may still be missing approximately $10 \%$ of the impedance in our model. 


\subsection{Impedances leading to multi-bunch instabilities}

Our attempts to identify the sources of coupled bunch instabilities were less successful, so far, than for the sources of single bunch instabilities. There is evidence from instability spectrum measurements at high energies of a source in the frequency range $900 \mathrm{MHz}$ to $1200 \mathrm{MHz}$ which could come from the next passband of the main $200 \mathrm{MHz}$ RF system. There is also some indication for the low Q, high frequency resonances in the pumping ports as being a possible source of multi-bunch instabilities at high energies.

The spectrum of the growing instability is in general broad band as mentioned before, and does not lend itself to easy interpretation. Methods to analyse these spectra are being investigated at the present time [13].

We have compiled a list of the known HOM's in the various RF cavities in the ring and have searched for evidence of excitation by direct observation in the cavities. At the moment we have 5 different types of cavities in the SPS. A résumé of the situation as known is given in Table 3.

\begin{tabular}{|c|c|c|}
\hline Source & Freq. range $(\mathrm{GHz})$ & Comment \\
\hline HOM in RF cavities & $0.1-1.109$ & 25 long. modes damped \\
& $0.286-1.14$ & 25 trans. modes damped \\
& $0.912-2.78$ & 13 modes seen, undamped \\
\hline Pumping ports & $1.39-4.23$ & Max. long. $\mathrm{R}_{\text {sh }} / \mathrm{Q}$ at 1.55, \\
& & $1.91,2.14,2.96 \mathrm{GHz}$ \\
\hline $400 \mathrm{MHz}$ band, (septa) & $0.343-0.49$ & 16 septa installed now, 8 later \\
\hline
\end{tabular}

Table 3: Known impedance sources

For the transverse plane the resistive wall impedance and its sources are well known. High frequency transverse modes in the cavities have been measured, see Table 3. The pumping ports also have transverse modes which were calculated but for which we do not at the moment expect problems.

\section{Reducing the machine impedance}

\subsection{Elements with high $\mathrm{R}_{\mathrm{sh}} / \mathrm{Q}$}

\subsubsection{Pumping ports}

The SPS machine designed in the early seventies has a FODO type lattice, the bending and focussing functions being separated in different magnets. There are six long straight sections each assigned to a different function, injection, extraction, RF, etc...; and 216 short straight sections, adjacent to quadrupoles, containing corrector magnets, position pick ups, and other instrumentation [14]. There are five main vacuum chamber types, but different cross-sections are found in the instrumentation, etc. The result is an extremely large number of abrupt changes in vacuum pipe dimension. In a significant number of cases, the different elements are connected by the insertion of a pumping port. This is in two halves, connected by a vacuum joint, one half being a solid cylinder, the other a bellows. Unfortunately this item has a larger diameter than any vacuum chamber and creates an RF cavity with, as we have seen, significant $R_{\mathrm{sh}} / \mathrm{Q}$. RF screens are being designed to lower the impedance of these $\sim 1000$ items [15].

The design of this screen must fulfil many difficult requirements:

- It must be easy to install and align. It will be necessary to remove one out of two magnets, $\sim 300$, to obtain access.

- A mechanism must be provided so that the RF contacts in the centre can be retracted and the shield divided in two, when and if a magnet must be removed or replaced.

- The pumping-down time for the machine and the residual pressure should not change significantly. 


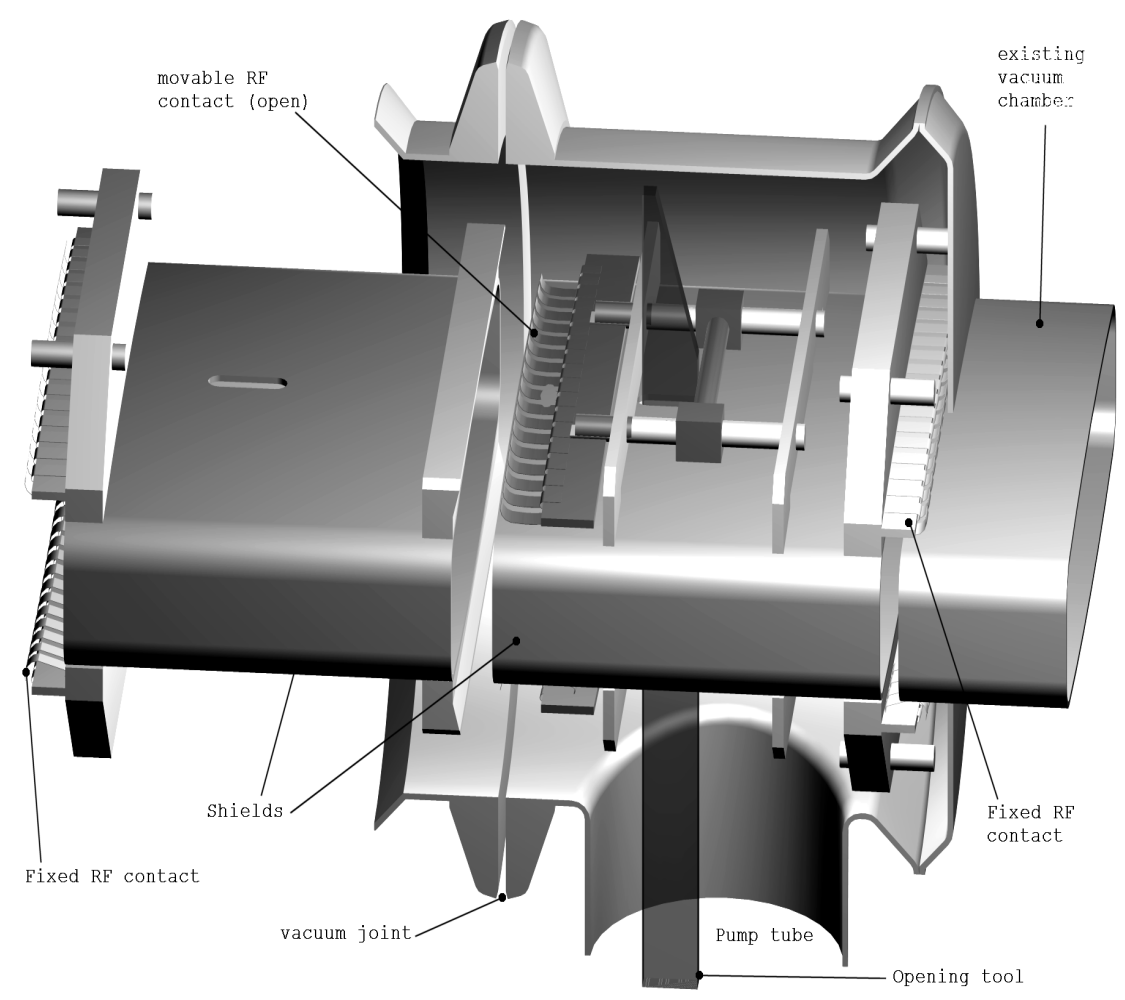

Figure 3: Pumping port screening, cut-away view.

- The shielding at $\mathrm{GHz}$ frequencies must be good.

Fig. 3 gives a 3-d cut-away view of the present design.

Critical points are:

- The RF contacts at each end, which are near the uneven weld between the vacuum chamber and the end flange.

- The pumping holes which must be large enough for pumping while not affecting screening. Keeping the holes towards the side is optimum for the longitudinal modes. Care must be taken with their position so as not to aggravate any of the transverse modes.

- The RF contact in the centre must be retractable. The mechanism to do this is by inserting a tool from beneath through slots in the vacuum chamber, pushing the contacts back. This contact must also be sufficiently flexible to take up significant movement, vertical and horizontal, $\sim \pm 2 \mathrm{~mm}$, during alignment and also position and angle changes when installed at the ends of bending magnets.

- Note also the two resistors, aluminium oxide on ceramic, which are inserted to lower the Q of any remaining resonances in the outer chamber.

Prototypes are being manufactured and will be installed in the machine in late summer 1999 for tests. The final installation of these screens will be spread over two long shutdowns, 1999/2000 and 2000/2001, the latter being associated with the closure of LEP.

\subsubsection{Septum screens}

The single bunch impedance measurement also identified the magnetic septa vacuum chambers as a significant source of impedance. Here, complete screening of this large vacuum tank is not possible due to the presence of the septum magnet and we have opted for a screen of diameter $28 \mathrm{~cm}$, (the diameter of the adjacent vacuum chambers), covering half the circumference. A photo is shown in Fig.4. 


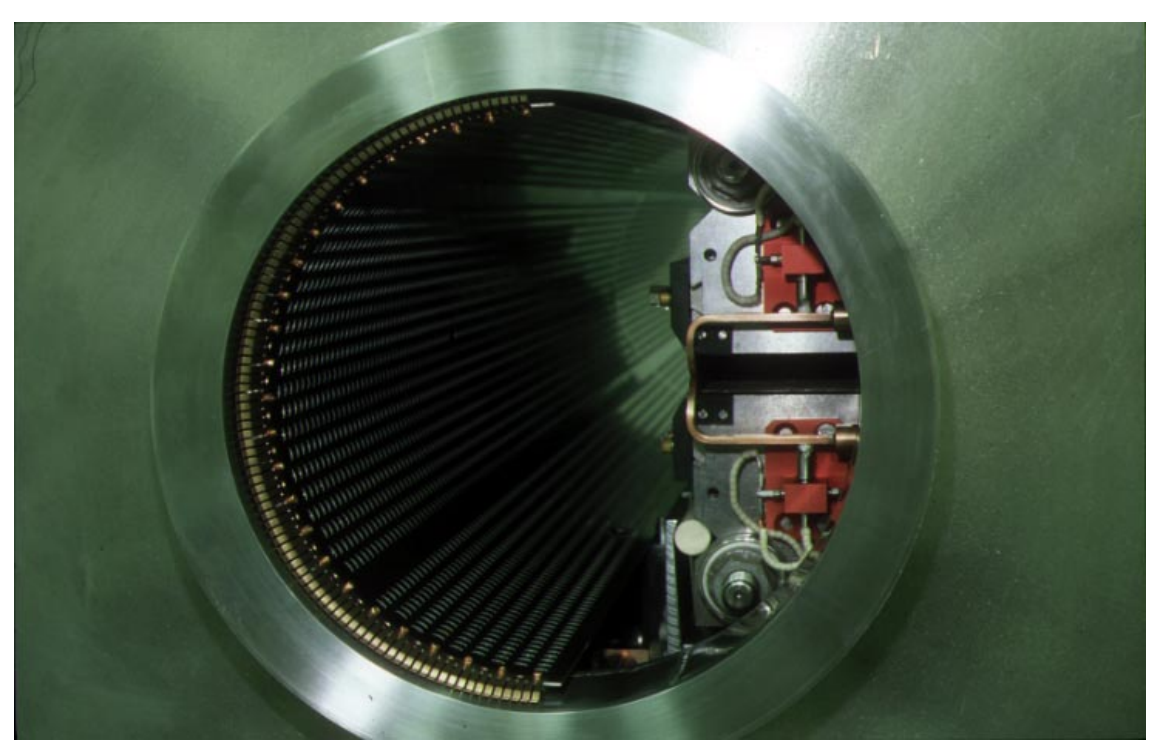

Figure 4: RF shield in the magnetic septum vacuum tank.

This is fitted along the length of the chamber, $2.67 \mathrm{~m}$ with RF contacts at each end. A steel sheet perforated with holes $6 \mathrm{~mm}$ in diameter is used. At each end two damping resistors are inserted, capacitively connected to the floating kicker element, to damp out resonances. Measurements in the laboratory have shown a reduction of $\geq 10 \mathrm{~dB}$ in impedance. A prototype was installed in the ring last year. Temperature measurements showed that heating due to beam image currents was negligible. This last shutdown half of the existing elements were shielded, the remainder will be shielded in the next.

\subsection{Elements with high $\mathrm{R}_{\mathrm{sh}}$}

For coupled bunch instabilities clearly the RF cavities are primary suspects in the search for devices with high $\mathrm{R}_{\text {sh }}$. However, the known HOMs up to $900 \mathrm{MHz}$ are already passively damped. If it is confirmed that the the next higher passband in the travelling wave cavities is a problem then we will examine ways to damp this. As mentioned before, there is also some evidence for the low Q, high frequency resonances in the pumping ports as being a possible source at high energies. Obviously screening will help here. When the LEP machine stops, the lepton acceleration systems in the SPS will be removed. This means the $100 \mathrm{MHz}$ and $200 \mathrm{MHz}$ standing wave cavity systems and the $352 \mathrm{MHz}$ superconducting cavity system - a total of 28 cavities in all. In addition we will remove all other elements like the wiggler, some instrumentation etc., dedicated to lepton acceleration, to clean the machine as much as possible. Nonetheless the possibility remains that there are other elements in the machine that may have significant impedance. Our search for sources will continue.

All these changes are aimed primarily at the longitudinal impedances, though the screening of objects and the removal of unnecessary equipment will help in the transverse plane as well.

\section{Other means of controlling the instabilities}

While trying as far as possible to reduce the impedance sources responsible for the different instabilities, we are also pursuing other methods to control the problem. For the single bunch instability one possibility is to raise the threshold by increasing the slip-factor $\eta$. This can be done by lowering the value of the transition energy. In the SPS with injection at $26 \mathrm{GeV}$ above and reasonably close to transition energy an improvement in threshold intensity by a factor 2.6 can be obtained by changing from $\gamma_{t r}=23.4$ (normal operation) to $\gamma_{t r}=19.5$. In principle either a dedicated set of quadrupoles would be installed to do this or the machine tunes could be significantly lowered. For tests [16] we have used 
the resonant behaviour of the dispersion function when operated at tune values close to a multiple of the machine superperiodicity of six. The result on the beam is measured by the unstable single bunch procedure outlined above. The results are given in Fig.5.

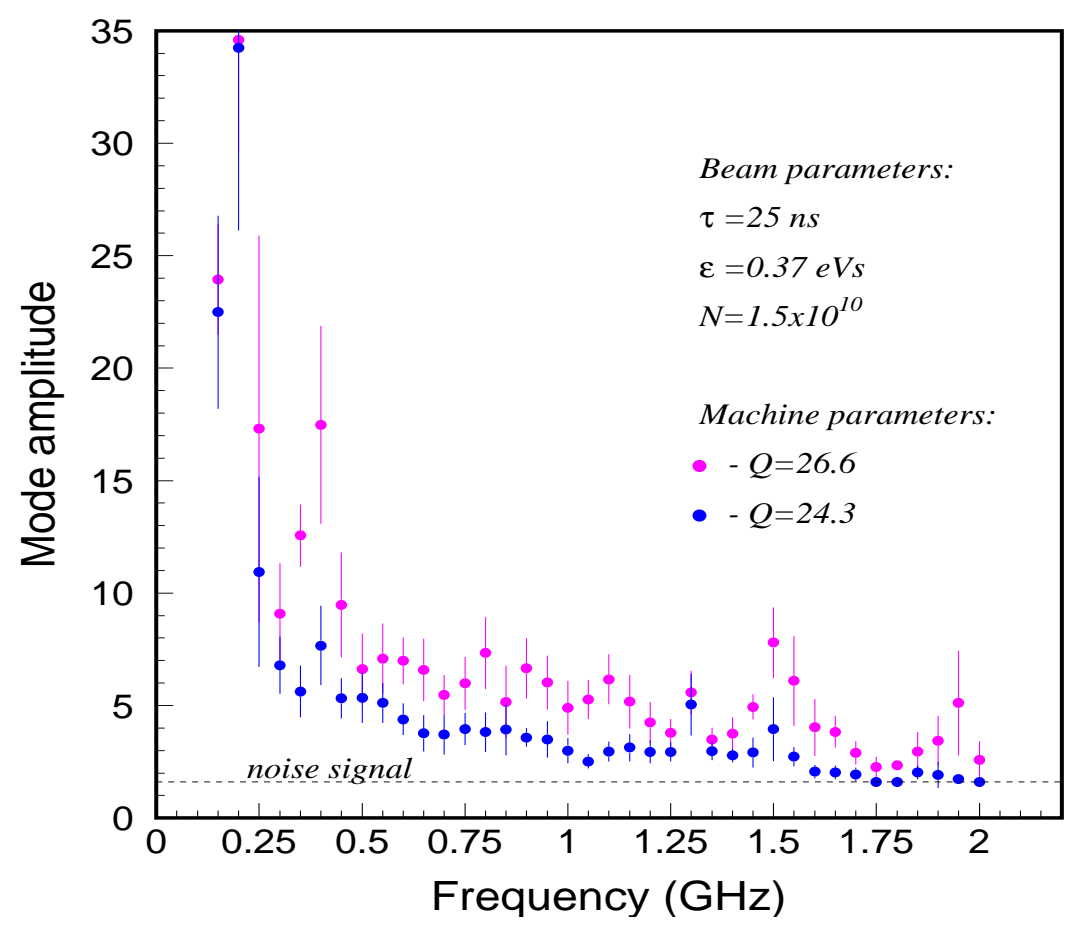

Figure 5: Spectrum of unstable modes for two values of $\gamma_{t r}$

This shows that the effect of the machine impedance on the beam is reduced by approximately a factor two for all frequencies except at $200 \mathrm{MHz}$, the fundamental RF frequency, and $1.3 \mathrm{GHz}$. Note that lowering the injection energy requires a larger matching voltage at injection which is beneficial for beam loading problems.

For multi-bunch problems, we are attacking on various fronts:

- Improved RF feedback around the main RF cavities. This is under construction.

- Feedforward on the main RF cavities. This is under construction.

- Landau damping using the existing $800 \mathrm{MHz}$ cavities. Analysis [17] has shown that the bunch shortening mode of operation (BS) is preferable to the bunch lengthening mode (BL) in practical applications. This is primarily due to the significantly reduced requirement on phase accuracy between the two RF systems in BS mode. This analysis confirms the operational experience of both HERA and the SPS where the BS mode is the only useful mode for Landau damping.

- Longitudinal feedback using recuperated lepton cavities. Preliminary designs are under way. Here the delicate problem is the coupling between the amplifier and cavity when bunch-by-bunch feedback ( $25 \mathrm{~ns}$ bunch spacing) is necessary.

Multi-bunch instabilities in the transverse plane are dominated by the resistive wall instability due to the vacuum chamber impedance, and the HOM modes in the RF cavities. The latter are already damped where known. The new transverse damper design will have a bandwidth extending to beyond $20 \mathrm{MHz}$, which will take care hopefully of all instabilities for the LHC type beams having a bunch spacing corresponding to $40 \mathrm{MHz}$ [8]. However it will not cover all modes possible with the Gran Sasso high intensity beam, where the bunch spacing corresponds to $200 \mathrm{MHz}$. Here we will have to rely on Landau damping from octupoles. The transverse emittance for this beam is however less critical. The main single bunch instability is the lowest mode head-tail and we hope to control this via the chromaticity. 


\section{Conclusion}

The future uses of the SPS accelerator at high intensities have created the need for an exciting programme of machine studies and hardware development projects. While standard techniques such as Landau damping and feedback are being explored to help control the beams, a vigorous programme to reduce the machine impedance, the source of the instabilities, is underway.

\section{Acknowledgements}

This report summarises the work of a team of people in the SL division. A large number is contributing to the design of the RF shields, in particular, F. Caspers, C. Dalmas, A. Rizzo, J. Ramillon and A. Spinks. I would like to thank E. Shaposhnikova for reading the paper and helpful comments.

\section{References}

[1] D. Boussard, G. Dome, T. Linnecar, A. Millich, Longitudinal Phenomena in the CERN SPS, IEEE Trans. on Nuclear Science, Vol. NS-24, No. 3, 1977

[2] D. Boussard, L. Evans, J. Gareyte, T. Linnecar, W. Mills, E.J.N. Wilson, Acceleration and Storage of a Dense Single Bunch in the CERN SPS, IEEE Trans. on Nuclear Science, Vol. NS-26, No. 3, 1979

[3] The SPS as Injector for LHC - Conceptual design, ed. P. Collier, CERN-SL-97-07 DI.

[4] J. Gareyte, Requirements of the LHC on its Injectors. Proc. workshop on LEP-SPS performance, Chamonix IX, Jan. 1999. CERN-SL-99-007 DI

[5] J. Tuckmantel, The SPS/LHC Longitudinal Interface. Proc. workshop on LEP-SPS performance, Chamonix IX, Jan. 1999. CERN-SL-99-007 DI

[6] E. Weisse, The CERN Neutrino Beam to Gran Sasso. Proc. workshop on LEP-SPS performance, Chamonix IX, Jan. 1999. CERN-SL-99-007 DI

[7] E. Shaposhnikova, Longitudinal Instabilities in the SPS. Proc. workshop on LEP-SPS performance, Chamonix IX, Jan. 1999. CERN-SL-99-007 DI

[8] W. Hofle, Towards a Transverse Feedback System and Damper for the SPS in the LHC Era. Particle Accelerators, 1997, Vol. 58, pp 269-279.

[9] T. Linnecar, E. Shaposhnikova, Microwave instability and Impedance Measurements in the CERN SPS. Particle Accelerators, 1997, Vol. 58, pp 241-255.

[10] T. Linnecar, E. Shaposhnikova, Another Method to Measure the Low-Frequency Machine Impedance. EPAC96, June 1996, Sitges.

[11] L. Vos, Computer Calculation of the Longitudinal Impedance of Cylindrically Symmetric Structures and its Application to the SPS. CERN SPS/86-21(MS).

[12] T. Bohl, T.P.R. Linnecar, E. Shaposhnikova, Measuring the Resonance Structure of Accelerator Impedance with Single Bunches. Phys. Rev. Letts. 1997, Vol. 78, No. 16.

[13] E. Shaposhnikova, Analysis of coupled bunch instability spectra. These proceedings.

[14] P. Collier, A.Spinks, Survey of the Short Straight Sections in the SPS for the Impedance Reduction Programme. CERN SL-Note-99-025 SLI.

[15] A. Spinks. Private communication. 
[16] T. Bohl, M. Lamont, T. Linnecar, W. Scandale, E. Shaposhnikova, Measurement of the Effect in Single Bunch Stability of Changing Transition Energy in the CERN SPS. EPAC98, June 1998, Stockholm, and CERN SL-98-024 RF.

[17] T. Bohl, T. Linnecar, E. Shaposhnikova, J. Tuckmantel, Study of Different Operating Modes of the 4th RF Harmonic Landau Damping System in the CERN SPS. EPAC98, June 1998, Stockholm, and CERN SL-98-026 RF. 